\title{
Modified Fair Queueing for Finite Buffer in ATM Networks
}

\author{
Arthur M. O. Lai, Danny H.K. Tsang \\ Department of Electrical \& Electronic Engineering \\ Hong Kong University of Science \& Technology \\ Clear Water Bay, Kowloon, Hong Kong \\ email: \{eearthur, eetsang\}@ee.ust.hk
}

\begin{abstract}
Service scheduling scheme that controls the order of servicing cells within an ATM node is very important in providing guaranteed services. Much attention of recent research have been paid on emulating the General Processor Sharing (GPS) system as closely as possible with low computational complexity. The primary motivation of emulating the GPS system is to provide traffic isolation and thus to achieve a maximum delay bound. This delay bound is guaranteed by guaranteeing the minimum bandwidth. However, after guaranteeing the minimum bandwidth, some excess bandwidth might be leftover and we argue that it should be used more intelligently to improve other system performance. In this paper, we propose a novel scheduling scheme that guarantees each traffic stream a minimum bandwidth while achieving a low cell loss probability in a finite buffer ATM node.
\end{abstract}

\section{INTRODUCTION}

Future ATM networks will support multiple service classes such as realtime service, non-realtime service, best-effort service and others. The success of ATM networks will depend on their ability to guarantee and deliver different quality of service (QoS) to support different classes of applications. The most common QoS parameters that need to be guaranteed are the cell transfer delay and the cell loss probability. In order to guarantee these QoS parameters, service scheduling scheme that controls the order of servicing the cells from different input traffic streams is needed within an ATM node. Proper choice of service scheduling scheme is very important in controlling the delay distribution of individual stream and preventing buffer overflow in a finite buffer ATM node.

There are two different objectives for any service scheduling scheme[1]: isolation and sharing. Isolation is to protect well-behaving traffic streams from those misbehaving traffic streams. Sharing, very different from isolation, is to improve system performance by sharing the system resources. Most of the previous research have focused on achieving only one, but not both of these objectives. For example, Earliest Deadline First (EDF) scheme and its variants are very effective sharing schemes, but they do not provide adequate isolation. Therefore, they have a good delay performance but well-behaving sources are easily affected by mis-behaving sources. On the other hand, Fair-Queueing systems like Packetised Generalized Processor Sharing (PGPS) and Weighted Fair Queueing (WFQ) are very effective methods for isolation. They provide guaranteed service for well-behaving sources and quarantine mis-behaving sources. However, they cannot share the network resources effectively among traffic streams and thus lead to suboptimal system performance.

Fair-Queueing system has a desirable property that it can guarantee a minimum bit rate for a traffic stream under any traffic conditions, i.e. provide isolation. It is also shown that guaranteeing this minimum bit rate is equivalent to providing a maximum delay bound for the traffic stream. In [6], a worst case delay bound of PGPS scheme is derived for a traffic stream that conforms to a leaky bucket filter. Most of the research have focused on designing a fair-queueing system which emulates the GPS system as closely as possible with low computational complexity. However, we question the necessity of emulating the GPS system since the primary objective for using fair queueing system within an ATM node is to provide a worst case delay bound for leaky bucket controlled sources. This delay bound can be guaranteed if the scheduler isolates the streams so that the minimum share of bandwidth for each stream can be guaranteed. In GPS system, whenever one or more streams is not backlogged, the unused bandwidth of these non-backlogged streams, which will be referred to as excess bandwidth in the rest of the paper, will be distributed to those backlogged streams in proportion to their guaranteed minimum shares. However, the bandwidth shares only reflect the long term requirements and redistributing the excess bandwidth this way does not further improve the delay bound. Therefore we suggest that we should share the excess bandwidth in a way such that it can reflect the instantaneous needs for the backlogged streams so that we can have a better balance between isolation and sharing. In a finite buffer per-stream queueing 
ATM node, each input stream will have it own buffer. Temporary overloading is very likely to occur when some of the streams simultaneously require high bit rate. During the temporary overloading, long queues will build up at some buffer and lead to overflow. Therefore we suggest that we should share the excess bandwidth in a more intelligent way in order to help the network to handle this short-term overloading more gracefully.

In this paper, we propose a novel service scheduling scheme called Modified Fair Queueing for Finite Buffer (MFQ-FB) which can isolate the mis-behaving streams to guarantee a minimum bandwidth for each stream, and at the same time can share the excess bandwidth to minimize the chance of buffer overflow. Our scheme can guarantee a maximum delay bound which is comparable to other proposed fair-queueing schemes. The service scheduling scheme is simple and requires a computational complexity of $O(1)$ only. In addition, we can provide a much lower cell loss probability than PGPS in an ATM node with finite buffer. The organization of this paper is as follows: In section 2, we present the network node model and describe our proposed scheduling scheme in detail. In section 3, we provide numerical results and some discussions on the results. Finally, we give the conclusion in section 4 .

\section{THE MODIFIED FAIR QUEUEING FOR FINITE BUFFER (MFQ-FB) SCHEME}

The ATM node we consider is modeled as a multiplexer fed by a superposition of $N$ traffic streams sharing a transmission link of capacity $C$. Cells from different streams have the same fixed size and thus the service time is the same for all the cells, called time slot. The multiplexer segregates the incoming traffic streams in a per-stream queueing model. Each incoming stream has it own physical buffer of finite size and a cell is discarded when the buffer is full upon its arrival. Each traffic stream $i, i=1,2, \ldots, N$, is associated with two separate counters $K_{i}, Q_{i}$ and a bandwidth share $S_{i}$. Counter $K_{i}$ keeps track of the credit of each queue and counter $Q_{i}$ keep track of the queue lengths. According to the value of the counters, the MFQFB scheduler selects the next head of line (HOL) cell to be transmitted at the end of the current transmission.

The idea of the MFQ-FB scheme is to provide a minimum bandwidth to individual traffic streams, and at the same time to avoid overflow of buffers in a finite buffer ATM node. In the MFQ-FB scheme, two individual servers are incorporated to determine the order of service for the cells. The Guaranteed Rate Server (GRS) is used to allocate the guaranteed minimum bandwidth to all the traffic streams according to their bandwidth shares. While the Overflow Protection Server (OPS), which makes use of the excess bandwidth left behind by the non-backlogging sessions, attempts to reduce the chance of buffer overflow

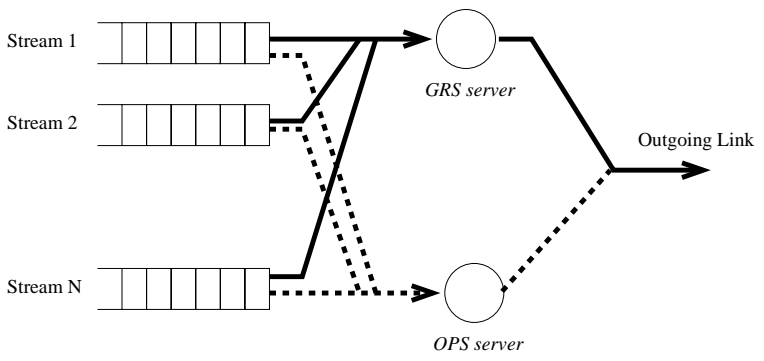

Fig. 1. The Switching node model

as much as possible. The conceptual view of the MFQ-FB scheme is shown in Fig. 1.

When all the traffic streams are backlogged, only the GRS server will be operating and it will distribute bandwidth to the streams according to their shares. However, in most of the cases, due to the bursty nature of traffic sources, the traffic sources are not likely to be active at the same time. Therefore the loading of the buffers will fluctuate with time and will be different for different streams, i.e. at some instants, some of the queues may be heavily loaded while the others may have a few cells only or may even become non-backlogged. In these cases, the GRS server will find that some of the queues are eligible for service but they are non-backlogged. The OPS server will then be triggered and reschedule these time slots to those backlogging queues according to their queue lengths. In this way, we can utilize the excess bandwidth to keep the queue lengths short and thus lower the chance of buffer overflow.

\subsection{The MFQ-FB Algorithm}

We define a time slot as the time interval needed for transmitting one cell by using the link capacity $C$. Here $S_{i}, i=1,2, \ldots, N$, denotes the fraction of the link capacity reserved for stream $i$ and $Q_{i}, i=1,2, \ldots, N$, denotes the queue length of stream $i$. On cell arrival at queue $i$, the counter $Q_{i}$ will be increased by one if the buffer is not full yet. Otherwise, $Q_{i}$ will not be updated and the cell will be discarded and considered to be lost.

1. Initialize: At the beginning of operation, set all the counters to zero

i.e. $K_{i}=0, i=1,2, \ldots, N$

2. Find the largest share among all the queues and denote it by $S_{\max }$ (i.e., $S_{\max }=\max \left\{S_{i}: i=\right.$ $1,2, \ldots, N\})$

3. Loop 1: $K_{i} \leftarrow K_{i}+\frac{S_{i}}{S_{\max }}$ for all $i, i=1,2, \ldots, N$

4. Let $\mathcal{K}=\left\{i: K_{i} \geq 1\right\}$

5. Loop 2: While $\mathcal{K}$ is non-empty 
6. Let $\mathcal{J}=\left\{j_{1}, \ldots, j_{k}\right\}$ and $|\mathcal{J}|=k$ such that Queues $j_{1}, \ldots, j_{k}$ are backlogged

and $Q_{j_{1}}=\max \left\{Q_{i}: i=j_{1}, j_{2}, \ldots, j_{k}\right\}$

7. Select one queue $i \in \mathcal{K}$

IF queue $i \in \mathcal{J}$

Serve one cell from queue $i$

ELSE

$Q_{i} \leftarrow Q_{i}-1$

Serve one cell from queue $j_{1}$

$Q_{j_{1}} \leftarrow Q_{j_{1}}-1$

8. $K_{i} \leftarrow K_{i}-1$

$\mathcal{K} \leftarrow \mathcal{K} \backslash\{i\}$

End Loop 2 (Goto step5)

\section{End Loop 1 (Goto step3)}

Here we give a brief description of our algorithm. The counter $K_{i}$ is used to store the number of transmission credits and they are all initialized to zero at the beginning. Based on the bandwidth shares, these counters are updated at the beginning of every cycle. Based on the value of the counters, the algorithm picks the queues with credits greater than or equal to one and forms a set $\mathcal{K}$. The queues in the set $\mathcal{K}$ are all eligible for service. The algorithm then picks the queues one by one and checks if it is backlogged. If this queue is backlogged, the algorithm picks the HOL cell from this queue and transmits it. Otherwise, the algorithm picks a HOL cell from the longest queue among the backlogged queues and transmits it. Each time a cell is transmitted, the counter of the queue that is supposed to receive service is decremented by one and that queue is removed from $\mathcal{K}$. These operations repeat until all the elements in the set $\mathcal{K}$ are removed. As the set $\mathcal{K}$ becomes empty, we will start another cycle by updating the counters according to their bandwidth shares.

\subsection{End-to-End Delay Bound}

To show the worst case performance of the MFQ-FB scheme, we only need to consider the worst case scenario where all the queues are backlogged. Because only in this case, all the streams are competing for bandwidth. If we can show that the MFQ-FB scheme can guarantee the minimum throughput for all the traffic streams when all the queues are backlogged, we can obtain the worst case end-to-end delay bound since each traffic stream can get more than the minimum bandwidth in other cases. It can easily be seen that when all the queues are backlogged, only the GRS server is operating and the operations become the same as CBFQ[7]. From [7], we can obtain the end-to-end delay bound for the MFQ-FB scheme and the bound is shown as follows.

Assume that all the traffic streams are leaky-bucket policed. If on the path of flow $i$, there are $k$ nodes in series and the scheduling scheme at each node is MFQ-FB, then the following bound on the end-to-end delay $D_{i}^{n}$ experienced by the $n$th cell from stream $i$ is satisfied:

$$
D_{i}^{n} \leq \frac{\sigma_{i}+(k-1) L}{\rho_{i}}+\sum_{j=1}^{k}\left(\frac{N^{j}-1}{C^{j}}+\frac{2}{S_{\max }^{j}}\right)+\sum_{j=1}^{k-1} \tau_{j}
$$

where $\sigma_{i}$ is the token pool size and $\rho_{i}$ is the token generation rate of the leaky-bucket filter of class $i . N^{j}$ is the number of flows served by node $j, C^{j}$ is the channel capacity of node $j$, and $S_{\text {max }}^{j}$ is the maximum bandwidth share of the flows at node $j . \tau_{j}$ is the propagation delay from node $j$ to node $j+1$.

\subsection{Computational Complexity}

In the MFQ-FB scheme, when a cell is selected for service, all the operations executed are $O(1)$. In step 3, the counters are independent and thus can be updated in parallel by small dedicated hardware. This leads to an $O(1)$ complexity for the counter update operation. In step 4 of the algorithm, the elements of the set $\mathcal{K}$ are not sorted according to their counters' values, which means the construction of $\mathcal{K}$ is done only by comparing the values of the counters to 1 . This can be practically implemented by using simple devices such as logic gates, performing only $O(1)$ operations. Also, in step 6, the maximum can be found easily by simple hardware with $O(1)$ complexity. All the other operations are $O(1)$. The scheme thus requires an $O(1)$ complexity.

\section{NUMERICAL RESULTS}

In this section, we present a series of simulation results to evaluate the cell loss performance of the MFQ-FB scheme and compare it with the PGPS scheme under different traffic conditions and buffer sizes using discrete-time simulations (where each time slot represents the service time of a cell). The sources of real-time traffic are modelled as two-state Markov processes. In each burst period, a geometrically distributed random number of cells are generated at some peak rate $P$ and the average burst size is $B$. After the burst has been generated, the source remains idle for some exponentially distributed random time with an average length of $I$. The average rate of cell generation $A$ is therefore given by $A^{-1}=\frac{I}{B}+\frac{1}{P}$. Each traffic source is then subject to an $(\sigma, \rho)$ leaky-bucket filter, where $\rho$ denotes the token generation rate and $\sigma$ denotes the token pool size. Any cell passes through the filter will consume a token and a cell can only pass through the filter only if 
there are tokens in the token pool. Whenever a cell enters the leaky-bucket filter and no token is available, it is considered to be nonconforming and will be dropped. In our simulations, 10 sources are connected to an outgoing link, which has bandwidth of $1000 \mathrm{cell} / \mathrm{s}$ and thus the cell service time is $1 \mathrm{~ms}$, i.e. each time slot is $1 \mathrm{~ms}$.

We fix the mean burst size to 20 cells, but the mean idle period and the peak cell rate are changed so as to adjust the loading. The burstiness, defined as the ratio of the peak cell rate to the mean cell rate, is kept constant when we vary the loading. Here the loading is defined as the sum of the average traffic offered to the node divided by the channel capacity. In the homogeneous case, the average traffic offered by all the 10 sources are equal. In the heterogeneous case, the average traffic offered is proportional to the bandwidth share. For the leaky-bucket filter, the token generation rate is equal to the average cell generation rate and the token pool size is equal to ten times the average burst size.

\subsection{Buffer Requirements under Various Loading}

First, we investigate the buffer requirements under different loading in order to achieve a certain cell loss probability. The results are shown in Fig. 2.

In Fig.2, the MFQ-FB scheme always requires smaller buffer size than the PGPS scheme to achieve the same cell loss probability $\left(10^{-3}\right.$ and $10^{-4}$ shown in Fig. 2). To show more clearly the improvement of the MFQ-FB scheme over the PGPS scheme, we plot the relative improvement in Fig. 3. Where the relative improvement is defined as the reduction in buffer size required by the MFQ-FB scheme over the PGPS scheme.

When the loading is low, the relative improvement of the MFQ-FB scheme over the PGPS scheme is relatively small. However, the improvement increases as the loading increases. The reason is that the occupancies of the

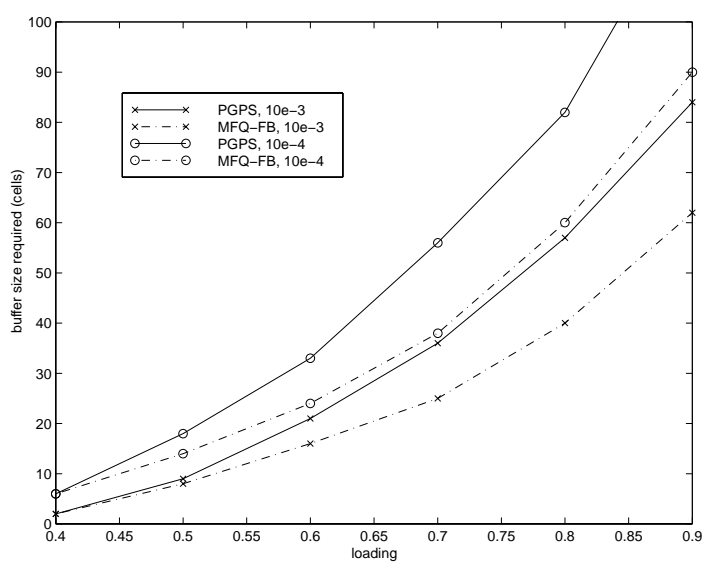

Fig. 2. Buffer Size Required vs. Loading

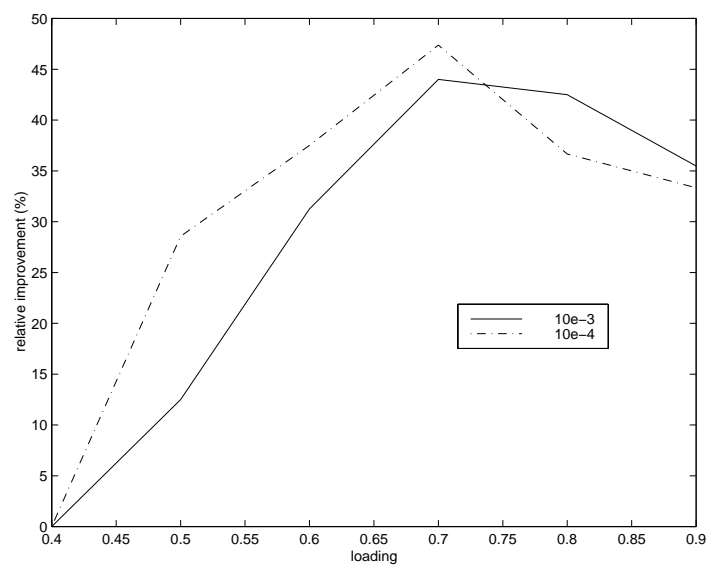

Fig. 3: Relative Improvement vs. Loading

buffers are usually low when the loading is low and therefore overflow is not likely to occur. Thus, the improvement of the MFQ-FB scheme is small when the loading is low. As the loading increases, there is a higher chance that some of the queues are being overloaded temporarily while other queues still have excess resources (both buffer and bandwidth) to help the overloading queues recover from the temporary overloading quickly and thus to reduce the chance of buffer overflow. Therefore the improvement of the MFQ-FB scheme increases as the loading increases. However when the loading is too high (e.g. above 0.9 ), the queues are very likely to be always backlogged and hence less excess bandwidth is available for the OPS server. Therefore the relative improvement decreases at heavy loading.

\subsection{Effect of Buffer Size on Cell Loss Probability}

\subsubsection{Homogeneous Traffic}

Next, we consider the homogeneous traffic condition where all the sources are statistically identical and have equal bandwidth shares. Since we have 10 sources in our simulations, the bandwidth shares are 0.1 for all sources.

In Figs. 4 and 5, the MFQ-FB scheme outperforms the PGPS scheme under both moderate and heavy loadings and different buffer sizes. The performance is very similar under both loading conditions. When the buffer size is very small, the cell loss is very high and the difference between the two schemes is also small. This is because when the buffer size is small, the buffer cannot hold all the incoming cells during a burst and therefore the MFQFB scheme can only offer little improvement even all the excess bandwidth is given to the overloading queues. As the buffer size increases, we can observe that the improvement of the MFQ-FQ scheme over the PGPS scheme also increases. In Fig. 4, the cell loss probability of the MFQFQ scheme is half of the PGPS scheme when the buffer size 


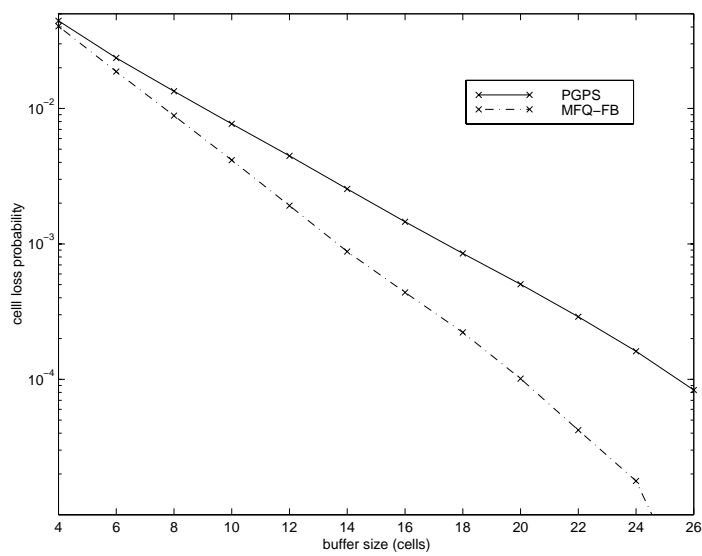

Fig. 4. Cell Loss Probability vs. Buffer Size Under Moderate Loading (homogeneous traffic, loading $=0.5$ )

is around 10, but when the buffer size is increased to 20, the cell loss probability of the MFQ-FB scheme is almost lower than that of the PGPS scheme by an order of magnitude. In other words, the relative improvement of the MFQ-FB scheme increases very quickly with buffer size. The reason is that when the buffer size increases, more and more incoming cells can be buffered during a burst and the MFQ-FQ scheme is able to help the overloading queues to recover quickly. The occupancy of the queues can be kept low and buffers are not likely to overflow when new bursts arrive. Therefore the cell loss probability can be reduced significantly by the MFQ-FB scheme.

\subsubsection{Heterogeneous Traffic}

The heterogeneous traffic environment is considered in this section. We keep the total number of sources to be 10 but divide them into two classes with different bandwidth shares. Each class has five sources. One class has shares $S_{1}=0.15$, the other class has shares $S_{2}=0.05$ and will be referred to as class 1 and class 2 respectively.

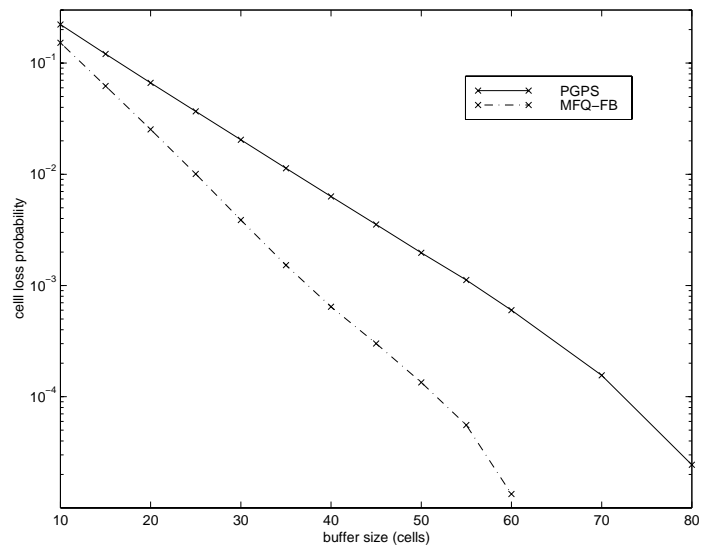

Fig. 5. Cell Loss Probability vs. Buffer Size Under Heavy Loading (homogeneous traffic, loading $=0.8$ )
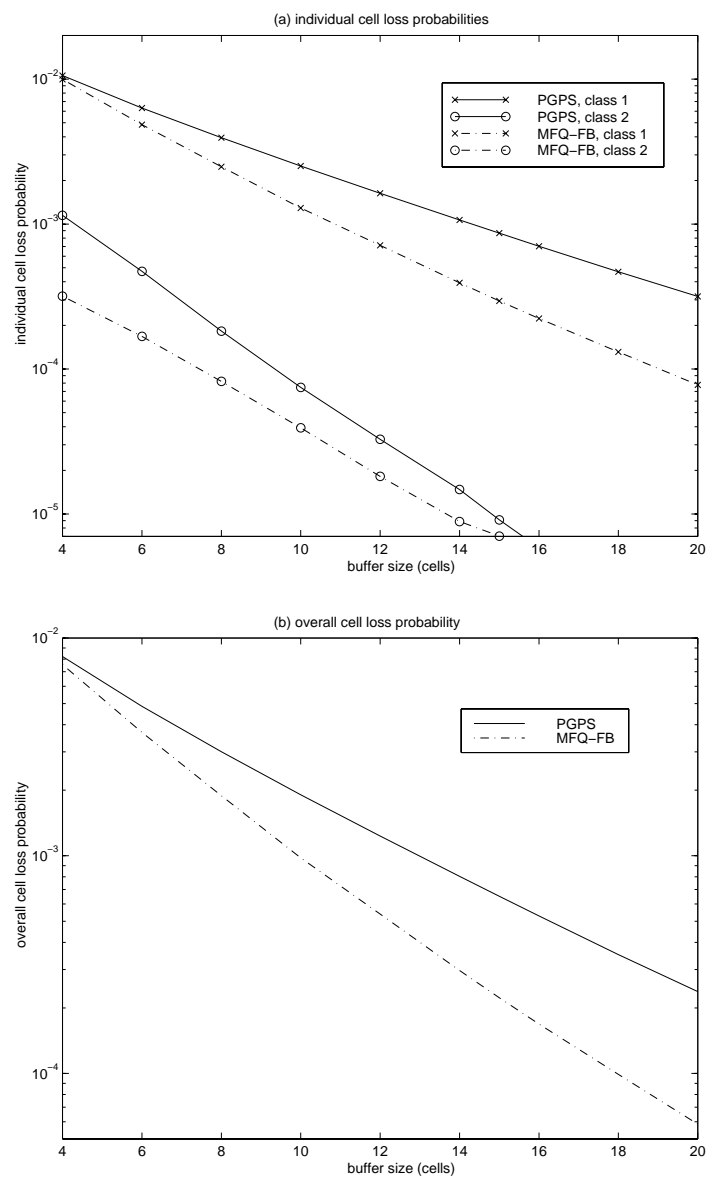

Fig. 6. Cell Loss Probability vs. Buffer Size Under Moderate Loading and Heterogeneous Traffic Condition (heterogeneous traffic, loading $=0.5$ )

In Fig. 6(a), the individual cell loss probabilities of the two classes are shown under different buffer sizes at moderate loading (0.5). For both classes, the MFQ-FB scheme always outperforms the PGPS scheme. For both schemes, the cell loss probability decreases as the buffer size increases. For class 1 traffic, the improvement of the MFQFB scheme over the PGPS scheme is small when the buffer size is small but it increases as the buffer size increases. The reason is similar to that in the homogeneous case. However for class 2 traffic, quite different from class 1 , the improvement decreases as the buffer size increases. The improvement is quite significant when the buffer size is small. This is because although the buffer size is small, the MFQ-FB scheme can still serve fast enough during a burst as the peak cell rate (during burst) is just $125 \mathrm{cell} / \mathrm{s}$ while the channel capacity is 1000 cell/s. Another reason is that when the loading is low or moderate, more excess bandwidth is available as some of the queues are more likely to be idle temporarily. As the buffer size increases, more cells can be held in the buffer during an active burst and the queues can recover quickly enough without utilizing the excess bandwidth. Therefore the improvement of 

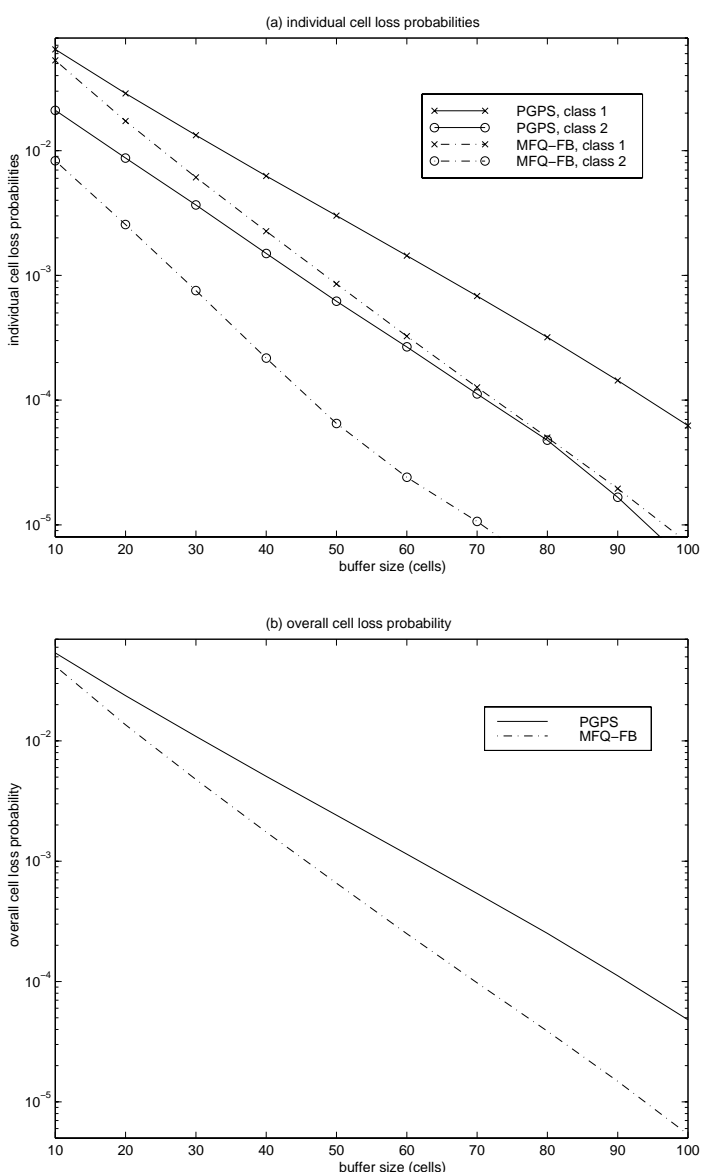

Fig. 7. Cell Loss Probability vs. Buffer Size Under Heavy Loading and Heterogeneous Traffic Condition

(heterogeneous traffic, loading $=0.8$ )

the MFQ-FB scheme decreases rather than increases when the buffer size increases. In Fig. 6(b), the overall cell loss probability of the two classes is shown. The MFQ-FB scheme clearly always outperforms the PGPS scheme. The behaviour is very similar to the homogeneous case. The overall cell loss probability decreases much more quickly for the MFQ-FB scheme and thus a much smaller buffer is needed for the MFQ-FB scheme to achieve the same overall cell loss probability as the buffer size increases. For example, with a buffer size of 6 , the MFQ-FB scheme has a very similar performance as the PGPS scheme (just a few percents improvement). But when the buffer size increases to 20 , the improvement becomes almost 50 percents over the PGPS scheme.

We now proceed to the heavy loading case(0.8) as shown in Fig. 7. First we discuss the individual cell loss probabilities. The situation is very similar to the moderate loading case for class 1 traffic. But for class 2 traffic, the performance is different from that under moderate loading. Since the peak cell rate (during burst) is not as low as in the moderate loading case and the occupancy of the queues are relatively high, the queues cannot recover quick enough even using the excess bandwidth when the buffer size is small. As the buffer size increases, more cells can be held in the buffer during a burst and the MFQ-FB scheme can help the queues recover from overloading more quickly. Hence, the improvement also increases. For the overall cell loss probability, the MFQ-FB again outperforms the PGPS scheme under different buffer sizes. The situation is also similar to the moderate loading case and the improvement increases as the buffer size increases.

\section{CONCLUSIONS}

In this paper, we have proposed a simple and effective service scheduling scheme, Modified Fair Queueing for Finite Buffer (MFQ-FB), which is able to provide an end-to-end delay bound for cell transfer delay with a lower buffer requirement. It guarantees a minimum throughput for each traffic stream by using the GRS Sever and reduces the cell loss due to buffer overflow by introducing the OPS Server. By guaranteeing the minimum throughput, our algorithm can provide an end-to-end delay bound. The algorithm has an implementation complexity of $O(1)$. Through simulations, we have shown that for a given buffer size, the MFQ-FB scheme can achieve a much lower cell loss probability than the PGPS scheme. In addition, we observe a significant improvement in buffer requirement over the normal operating region of networks.

\section{REFERENCES}

[1] D. Clark, S. Shenker and L. Zhang. Supporting Real-time Application in an Integrated Services Packet Network. Proceedings of ACM SIGCOMM 1992, pp.14-23.

[2] S.J. Golestani. A Self-Clocked Fair Queueing Scheme for Broadband Applications. Proceedings of INFOCOM'94, pp.636646, 1994.

[3] D.S. Lee. Weighted Longest Queue First: An Adaptive Scheduling Discipline for ATM Networks. Proceedings of IEEE INFOCOM'97, pp.318-325, 1997.

[4] H. Saito. Optimal Queueing Discipline for Real-Time Traffic at ATM Switching Nodes. IEEE Transactions on Communications, Vol. 38, No. 12, Dec. 1990.

[5] A.K. Parekh, R.G. Gallagher. A Generalized Processor Sharing Approach to Flow Control in Integrated Services Networks. in Proceedings of IEEE INFOCOM'92, pp.915-924.

[6] A. Demers, S. Keshav and S. Shemker. Analysis and Simulation of a Fair Queueing Algorithm. Proceedings of SIGCOMM'89, Sept 1989 .

[7] B. Bensaou, K.T. Chan, D.H.K. Tsang. Credit-Based Fair Queueing (CBFQ): A Simple and Feasible Scheduling Algorithm for Packet Networks. IEEE ATM'97 Workshop Proceedings, pp.589-594, 1997. 